\title{
Linear and nonlinear effect of sheared plasma flow on resistive tearing modes
}

\author{
Qiming $\mathrm{Hu}^{1}{ }^{1,}$ ), $\mathrm{Q} . \mathrm{Yu}^{2}$, and Xiwei $\mathrm{Hu}^{1}$ \\ ${ }^{1}$ State Key Laboratory of Advanced Electromagnetic Engineering and Technology, School of \\ Electric and Electronic Engineering, Huazhong University of Science and Technology, 430074, \\ Wuhan, China \\ ${ }^{2}$ Max-Planck-Institut für Plasmaphysik, 85748 Garching, Germany
}

\begin{abstract}
The effect of sheared plasma flow on the $m / n=2 / 1$ tearing mode is studied numerically ( $m$ and $n$ are the poloidal and toroidal mode numbers). It is found that in the linear phase the plasma flow with a weak or moderate shear plays a stabilizing effect on tearing mode. However, the mode is driven to be more unstable by sufficiently strong sheared flow when approaching the shear Alfvén resonance (AR). In the nonlinear phase, a moderate (strong) sheared flow leads to a smaller (larger) saturated island width. The stabilization of tearing modes by moderate shear plasma flow is enhanced for a larger plasma viscosity and a lower Alfvén velocity. It is also found that in the nonlinear phase AR accelerates the plasma rotation around the 2/1 rational surface but decelerates it at the AR location, and the radial location satisfying AR spreads inwards towards the magnetic axis.
\end{abstract}

\section{Introduction}

It is widely recognized that plasma flow may have considerable effect on plasma performance and MHD instabilities. ${ }^{1}$ A number of experimental studies have examined the effect of sheared plasma flow on tearing mode (TM). For both high- and low-aspect-ratio tokamaks, it is found that reducing plasma flow clearly decreases the stability of TM and makes pre-existing saturated islands larger. $^{2-4}$ It has also been found in DIII-D that flow shear has a stabilizing effect on the $m / n=3 / 2$ neoclassical tearing mode (NTM), ${ }^{5}$ where $m$ and $n$ are the poloidal and toroidal mode number. In addition, flow shear at the rational surface is shown to make the effective tearing stability index $\Delta^{\prime}$ more negative. 6,7

In the theoretical aspect, both analytical and numerical studies have been carried out. In the linear phase and in the slab geometry, plasma flow shear is found to either increase or decrease the linear growth rate, depending on the plasma viscosity, the magnetic shear and the strength of flow shear. 8 When the velocity shear is strong enough, it will give rise to short wave-length Kelvin-Helmholtz $(\mathrm{KH})$ instabilities ${ }^{10-12}$ and excitation of Alfvén resonances. ${ }^{13-15}$ In the nonlinear phase, the flow shear is found to decrease the saturated island width ${ }^{16}$ and leads to deformation of magnetic island. ${ }^{17}$ Recently, the effect of velocity profiles on both TM and NTMs has been investigated in toroidal geometry, ${ }^{18}$ and the differential rotation between rational surfaces is found to be stabilizing, while the toroidal velocity shear at the resonant surface is destabilizing. Besides, the effect of sheared plasma rotation on the stability of TM in an Ohmic regime is investigated. ${ }^{19}$ It is found that plasma rotation in the equivalent toroidal direction can result in either an increase or a decrease of the mode growth rate. As shown in Ref. 17, the velocity profile is crucial in determining the effect of flow shear on the tearing mode stability. However, most of the previous numerical results are obtained based on a narrow flow shear layer around the rational surface, which is substantially different from the actual rotation velocity profile of tokamak plasma. The effect of plasma shear flow on TMs remains to be an important topic for both existing tokamaks and for ITER. $^{20}$

In this work, the effect of sheared plasma flow on both the linear and nonlinear growth of the

a) Author to whom correspondence should be addressed. Electronic mail: huqm@ hust.edu.cn. 
$m / n=2 / 1 \mathrm{TM}$ is studied with the magnetic Reynold number $\mathrm{S}$ being relevant to tokamak plasmas $\left(\mathrm{S} \sim 10^{6 \sim 9}\right)$. In particular, we investigate the effect of poloidal sheared flow on the linear growth rate and the nonlinear saturation of 2/1 TM using the large aspect ratio and low $\beta$ approximation. In the next section, the theoretical model utilized in our numerical calculations is introduced. In Sec. III the numerical results are presented, including the effect of shear Alfvén resonance (AR). Finally, the major results are summarized in Sec. IV.

\section{Theoretical model}

The large aspect-ratio tokamak and low $\beta$ approximation is utilized. The magnetic field $\boldsymbol{B}$ is defined as

$$
\boldsymbol{B}=B_{0 t} \boldsymbol{e}_{t}-(k r / m) B_{0 t} \boldsymbol{e}_{\theta}+\nabla \psi \times \boldsymbol{e}_{t},
$$

where $\psi$ is the helical flux function, $m / r$ and $k=n / R$ are the wave vectors in the $\boldsymbol{e}_{\theta}$ (poloidal) and $\boldsymbol{e}_{t}$ (toroidal) directions, and $r$ and $R$ are the minor and the major radius, respectively, the subscript 0 denotes the equilibrium quantity.

Ohm's law and the equation of motion (after taking the operator $\boldsymbol{e}_{t} \cdot \nabla \times$ ),

$$
\begin{aligned}
& \frac{d \psi}{d t}=E_{0}-\eta j, \\
& \rho \frac{d}{d t} \nabla_{\perp}^{2} \phi=-B \nabla_{\|} j+\rho \mu_{\perp} \nabla_{\perp}^{4} \phi+S_{m},
\end{aligned}
$$

are utilized, where

$$
d / d t=\partial / \partial t+v \cdot \nabla
$$

$v$ is the plasma velocity,

$$
j=-\nabla^{2} \psi-2(n / m R) B_{0 t},
$$

is the toroidal plasma current density, $\eta$ is the plasma resistivity, $\rho$ the mass density, and $\mu$ the plasma viscosity. The stream function $\phi$ is defined by

$$
v=\nabla \phi \times \boldsymbol{e}_{t},
$$

and the subscripts $\|$ and $\perp$ stand for the parallel and the perpendicular components, respectively. $E_{0}$ is the equilibrium electric field for maintaining the original equilibrium plasma current density, and $S_{m}$ in equation (3) is the momentum source given by

$$
S_{m}=\Omega_{E}\left[1-(r / a)^{\alpha_{1}}\right]^{\alpha_{2}}
$$

which leads to a poloidal equilibrium plasma rotation.

Normalizing the length to the plasma minor radius $a$, the time $t$ to $\tau_{R}, \psi$ to $a B_{0 t}, \phi$ to $a^{2} / \tau_{R}$, velocities to $a / \tau_{R}, j$ to $B_{0 t} / a$, viscosity to $a^{2} / \tau_{R}$, and plasma resistivity $\eta$ to its value at the rational surface (RS), Eqs. (2) and (3) become

$$
\begin{aligned}
& \frac{d \psi}{d t}=E_{0}-\eta_{N} j, \\
& \frac{\mathrm{d} U}{\mathrm{~d} t}=-S^{2} \nabla_{\|} j+\mu_{N} \nabla_{\perp}^{2} U+S_{m},
\end{aligned}
$$

where $\tau_{R}=a^{2} \mu_{0} / \eta$ is the resistive time at RS, $\mathrm{S}=\tau_{\mathrm{R}} / \tau_{\mathrm{A}}$ the magnetic Reynolds number at $\mathrm{RS}, \tau_{\mathrm{A}}=$ $a / \mathrm{V}_{\mathrm{A}}$ the Alfvén time at $\mathrm{RS}, \mathrm{V}_{\mathrm{A}}=B_{0 t} /\left(\mu_{0} \rho\right)^{1 / 2}$ the Alfvén velocity at $\mathrm{RS}, \mathrm{U}=-\nabla_{\perp}^{2} \phi$ the plasma 
vorticity, $\eta_{N}$ the normalized plasma resistivity, and $\mu_{N}$ the normalized plasma viscosity.

$\psi$ and $v$ are expressed in terms of Fourier components of the form $F(r, \theta, \varphi)=F_{0}(r)+$ $\Sigma F_{j}(r) \cos \left(m_{j} \theta+n_{j} \varphi\right)$, where $m_{j}$ and $n_{j}$ are the poloidal and toroidal mode numbers of the $j^{\text {th }}$ component, and $\theta$ and $\varphi$ are the poloidal and toroidal angle, respectively.

The radial profile of the safety factor $q(r)=r B_{0 t} /\left(R B_{0 \theta}\right)$ is chosen to be the form

$$
q(r)=q_{0}\left(1+\left(r / r_{0}\right)^{2 p}\right)^{1 / p},
$$

where $q_{0}=0.95, r_{0}=0.55$ and $p=1.5$ are taken with the $q=2 / 1$ surface located $r_{s}=0.7 a$ and edge safety factor $q_{a}=3.5$, and the local magnetic shear length at $q=2$ surface $L_{q}=q /(a q)^{\prime}{ }_{\mathrm{rs}}=1.925$, based on the J-TEXT parameters. ${ }^{21,22}$
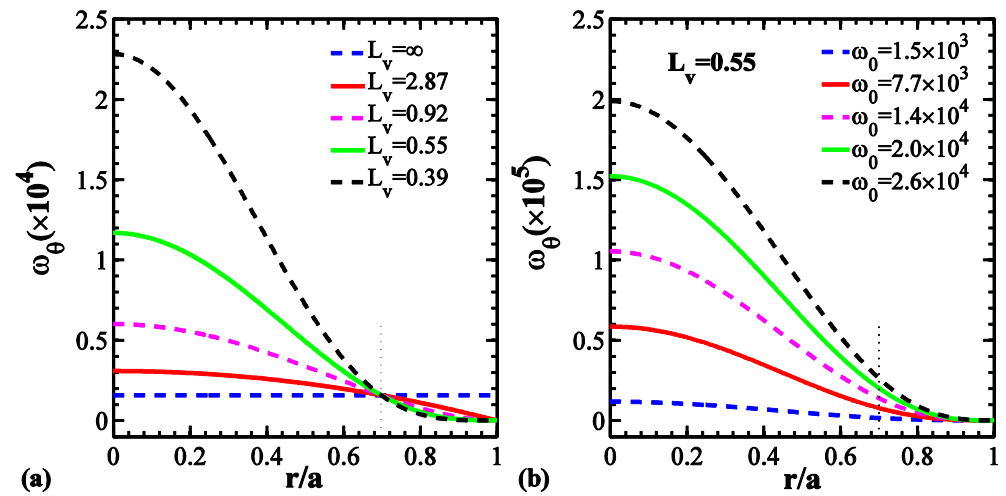

FIG. 1. (a) Radial profiles of the normalized poloidal angular rotation frequency $\omega_{\theta}$ with $\omega_{0}=1.6 \times 10^{3}$ for different $L_{\mathrm{v}}$, obtained by adjusting the values of $\Omega_{\mathrm{E}}, \alpha_{1}$ and $\alpha_{2}$ in Eq. (7). (b) Radial profiles of $\omega_{\theta}$ for $L_{\mathrm{v}}=0.55$ with different $\omega_{0}$. Here $\omega_{0}$ represents the poloidal angular frequency at the $2 / 1$ rational surface, and $L_{\mathrm{v}}=-v_{\theta} /\left(a v_{\theta}\right)^{\prime}$ rs is the flow shear length at $2 / 1$ rational surface.

The radial profiles of the normalized equilibrium poloidal angular rotation frequency $\omega_{\theta}$ utilized for our calculations are shown in Fig. 1. Fig. 1(a) shows profiles of $\omega_{\theta}$ for $\omega_{0}=\omega_{\theta}\left(r_{\mathrm{s}}\right)=$ $1.6 \times 10^{3}$ with different $L_{\mathrm{v}}$, obtained by adjusting the values of $\Omega_{\mathrm{E}}, \alpha_{1}$ and $\alpha_{2}$ in Eq. (7), where $L_{\mathrm{v}}$ is the dimensionless flow shear length defined as $L_{\mathrm{v}}=-v_{\theta} /\left(a v_{\theta}\right)^{\prime}{ }_{\mathrm{rs}}$. Here, $\omega_{0}$ represents the poloidal angular frequency at $q=2$ surface except mentioned elsewhere. A smaller value of $L_{\mathrm{v}}$ corresponds to stronger shear rate at the resonant surface, and $L_{\mathrm{v}}=\infty$ corresponds to rigid plasma rotation. Fig. 1(b) shows the radial profiles of $\omega_{\theta}$ for $L_{\mathrm{v}}=0.55$ with different $\omega_{0}$, obtained by just adjusting the values of $\Omega_{\mathrm{E}}$. The vertical dotted line at $r=0.7 a$ shows the radial location of $q=2$ rational surface.

Equations (8) and (9) are solved simultaneously using the initial value code TM1, which has been used for modeling the nonlinear growth and saturation of NTMs, their stabilization by RF current $^{23,24}$ and the effect of resonant magnetic perturbations (RMPs) on resistive TM earlier. ${ }^{21,22}$

\section{Numerical results}

In this section, numerical results of sheared plasma flow effects on TM stability are presented. A single $m / n=2 / 1$ magnetic island is considered here. For all these results, the resistive time is fixed, and the variation of the $S$ values corresponds to the change of Alfvén velocity

\section{A. Linear results}

Before studying the sheared plasma flow effects on tearing stability, it is helpful to first have a look at the effect of plasma viscosity on the linear mode growth rate, as shown in Fig. 2. In order to remove the effect contributed by flow shear, stationary plasma is chosen $\left(\omega_{\theta}(r)=0\right)$. Plasma viscosity is selected as $\mu_{N}=0.028,0.28,2.8,28$ and 280 , here $\mu_{N}=2.8$ corresponds to $1 \mathrm{~m}^{2} / \mathrm{s}$ (in anomalous transport level) for $a=0.27 \mathrm{~m}$ and $\tau_{R}=0.2 \mathrm{~s}$, and the ratio between $\tau_{R}$ and viscous time $\tau_{v}$ is $\tau_{R} / \tau_{v}=2.7$. Fig. 2 shows that for a small plasma viscosity (i.e. $\mu_{N}=0.028,0.28$ ), the linear 
growth rate $\gamma \tau_{\mathrm{A}}$ scales as $\mathrm{S}^{-3 / 5}$, being consistent with the scaling of the classical TM. ${ }^{25}$ Besides, the linear growth rate decreases with increasing plasma viscosity, indicating the stabilizing effect of plasma viscosity on $\mathrm{TM}^{9}{ }^{9}$ A sufficiently large plasma viscosity substantially decreases $\gamma \tau_{\mathrm{A}}$, and the linear growth rate increases with increasing $S$ when $S<4 \times 10^{6}\left(\mathrm{~S}<1 \times 10^{7}\right)$ for $\mu_{N}=28\left(\mu_{N}=280\right)$. In addition, the $2 / 1 \mathrm{TM}$ becomes stable for $\mathrm{S}<1.1 \times 10^{6}$ with $\mu_{N}=28$ and for $\mathrm{S}<3.5 \times 10^{6}$ with $\mu_{N}=$ 280. For a sufficiently large value of $S$, the linear growth rate is consistent with the classical TM scaling. Fig. 2 indicates that, on one hand, the plasma viscosity contributes to a stabilizing effect on TM stability. On the other hand, the stabilizing effect depends on $S$, and for a smaller $S$ the stabilizing effect is considerably stronger.

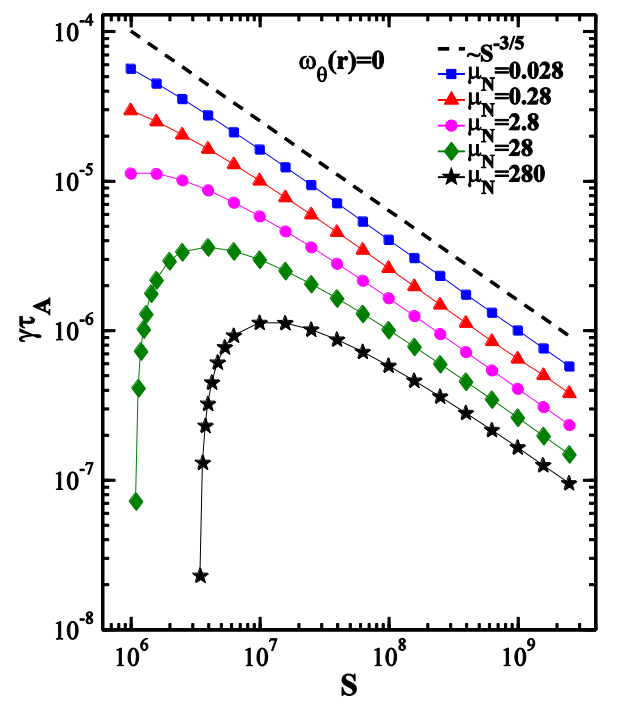

FIG. 2 Dependence of normalized linear growth rate $\gamma \tau_{\mathrm{A}}$ on magnetic Reynold number $\mathrm{S}$ for different plasma viscosity without plasma rotation. A sufficiently large plasma viscosity stabilizes TM for low $\mathrm{S}$ value.
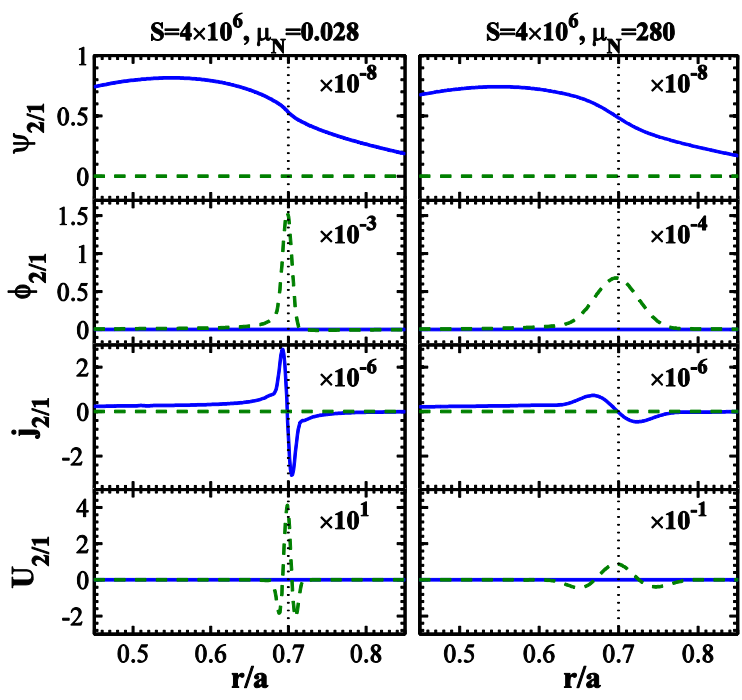

FIG. 3 Corresponding to Fig. 2, radial profiles of magnetic flux $\psi_{2 / 1}$, stream function $\phi_{2 / 1}$, current density $j_{2 / 1}$, and vorticity $U_{2 / 1}$ for $\mathrm{S}=4 \times 10^{6}, \mu_{N}=0.028$ (left side) and $\mu_{N}=$ 280 (right side). The solid (dash) curve represents the real (imaginary) part.

Corresponding to Fig.2, the magnetic flux $\psi_{2 / 1}$, stream function $\phi_{2 / 1}$, current density $j_{2 / 1}$, and vorticity $U_{2 / 1}$ are shown in Fig. 3 for $\mathrm{S}=4 \times 10^{6}, \mu_{N}=0.028$ (left) and $\mu_{N}=280$ (right), where the solid (dashed) curve represents the real (imaginary) part. The imaginary part of magnetic flux $\operatorname{Im}\left\{\psi_{2 / 1}\right\}=0$ and the real part of stream function $\operatorname{Re}\left\{\phi_{2 / 1}\right\}=0$, indicating $\phi_{2 / 1}$ have a phase advance of $\pi / 2$ relative to $\psi_{2 / 1}$, which is similar to the results of $m / n=1 / 1$ mode. ${ }^{12}$ For $\mu_{N}=0.028$ (left side), $j_{2 / 1}$ and $U_{2 / 1}$ are much more localized at the $2 / 1$ rational surface than those for $\mu_{N}=280$. With about the same amplitude of $\psi_{2 / 1}$, the amplitude of $j_{2 / 1}$ and $U_{2 / 1}$ are about one order smaller for $\mu_{N}=280$ than those for $\mu_{N}=0.028$.

Based on the results shown in Figs. 2 and 3, the sheared plasma flow effects on 2/1 TM are presented in the following. The plasma viscosity is selected to be $\mu_{N}=2.8$, which has been used in numerical modeling to quantitatively explain the experimental results observed in J-TEXT tokamak. $^{21,22}$ In Fig. 4(a) the linear growth rates are shown as a function of $\mathrm{S}$ for $L_{\mathrm{v}}=2.87$ with different $\omega_{0}$, and it is found that $\gamma \tau_{\mathrm{A}}$ decreases with increasing $\omega_{0}$ for $\mathrm{S}<10^{7}$. While for $\mathrm{S}>10^{7}, \gamma \tau_{\mathrm{A}}$ only decreases slightly and has the scaling $\mathrm{S}^{-3 / 5}$. For a stronger shear rate with $L_{\mathrm{v}}=0.55$, the results are shown in Fig. 4(b). Being different from the results shown in Fig. 4(a), in this case $\gamma \tau_{\mathrm{A}}$ becomes larger with increasing $\omega_{0}$ for $\mathrm{S}<2.5 \times 10^{6}$. When further increasing the shear rate to $L_{\mathrm{v}}=0.3$, the effect of sheared plasma flow on the linear growth rate becomes more complicated as shown in Fig. $4(\mathrm{c})$. For $\mathrm{S}<2 \times 10^{7}$, flow with moderate amplitude $\left(\omega_{0}<2.2 \times 10^{4}\right)$ destabilizes the $2 / 1 \mathrm{TM}$, and flow with strong enough amplitude $\left(\omega_{0} \geq 2.2 \times 10^{4}\right)$ makes the linear growth rate peak at a certain value of 
S. Taking the curve of $\omega_{0}=6 \times 10^{4}$ as an example, the peak point is at $\mathrm{S}=4 \times 10^{6}$. The $2 / 1 \mathrm{TM}$ becomes stable for $\mathrm{S}<3 \times 10^{6}$, and $\gamma \tau_{\mathrm{A}}$ increases with increasing $\mathrm{S}$ for $3 \times 10^{6}<\mathrm{S}<4 \times 10^{6}$ but decreases with increasing $\mathrm{S}$ for $\mathrm{S}>4 \times 10^{6}$. For $\mathrm{S}>2 \times 10^{7}$, the sheared plasma flow only contributes to a weak stabilizing effect on the $2 / 1 \mathrm{TM}$.
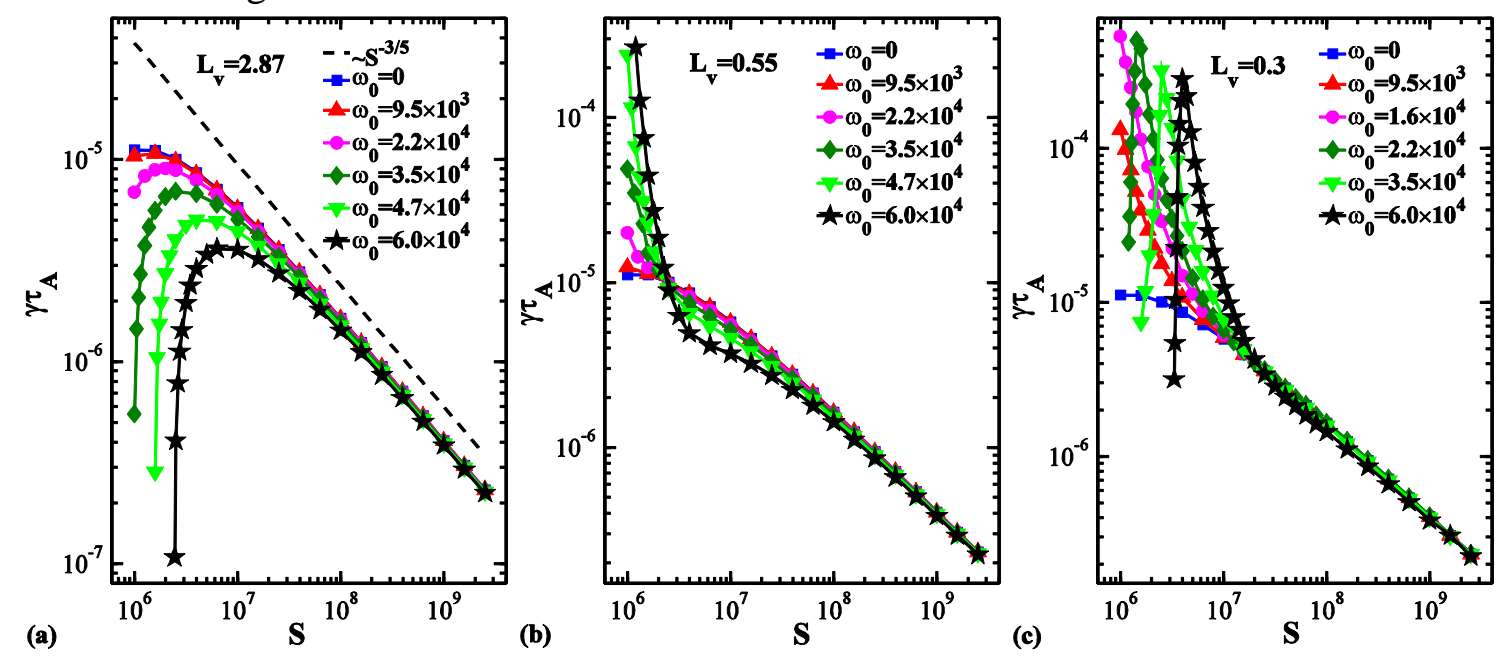

FIG. 4 Dependence of normalized linear growth rate $\gamma \tau_{\mathrm{A}}$ on magnetic Reynold number $\mathrm{S}$ for different $\omega_{0}$ and (a) $L_{\mathrm{v}}=$ 2.87, (b) $L_{\mathrm{v}}=0.55$ and (c) $L_{\mathrm{v}}=0.3$. For weak shear rate, the sheared plasma flow stabilizes the tearing stability, while for strong shear rate, the flow shear destabilizes tearing stability for low $\mathrm{S}$ value.

The results shown in Fig. 4 reveals that the plasma flow with a weak shear stabilizes the 2/1 TM, and faster flow enhances the stabilization effect. Such a stabilizing effect is more obvious for low $\mathrm{S}$ values. However, the plasma flow with a too strong shear rate mostly contributes to a destabilizing effect for low $S$ values but a weak stabilizing effect in large $S$ regime. In order to understand the opposite effects, corresponding to the case with $\omega_{0}=6 \times 10^{4}$ in Fig. 4(c), radial profiles of $\psi_{2 / 1}, \phi_{2 / 1}, j_{2 / 1}$, and $U_{2 / 1}$ for $\mathrm{S}=3 \times 10^{6}, \mathrm{~S}=4 \times 10^{6}, \mathrm{~S}=5.2 \times 10^{6}$ and $\mathrm{S}=5.8 \times 10^{6}$ are shown in Fig. 5. For $\mathrm{S}=3 \times 10^{6}$, it is found that except for the perturbation locating at the $q=2$ rational surface at $r=0.7 a$, there is another perturbation locating at $r=0.5 a$, and its amplitude is much larger than that at the $q=2$ surface, being different from the results as shown in Fig. 3 . For $\mathrm{S}=$ $4 \times 10^{6}$, similarly, there is another perturbation locating at $r=0.34 a$, and its amplitude is about one order of magnitude larger than that for $S=3 \times 10^{6}$. For $S=5.2 \times 10^{6}$, the additional perturbation locates at $r=0.14 a$, and its amplitude is much smaller than that of $\mathrm{S}=4 \times 10^{6}$. For $\mathrm{S}=5.8 \times 10^{6}$, there is no additional perturbation except that at the $q=2$ surface.
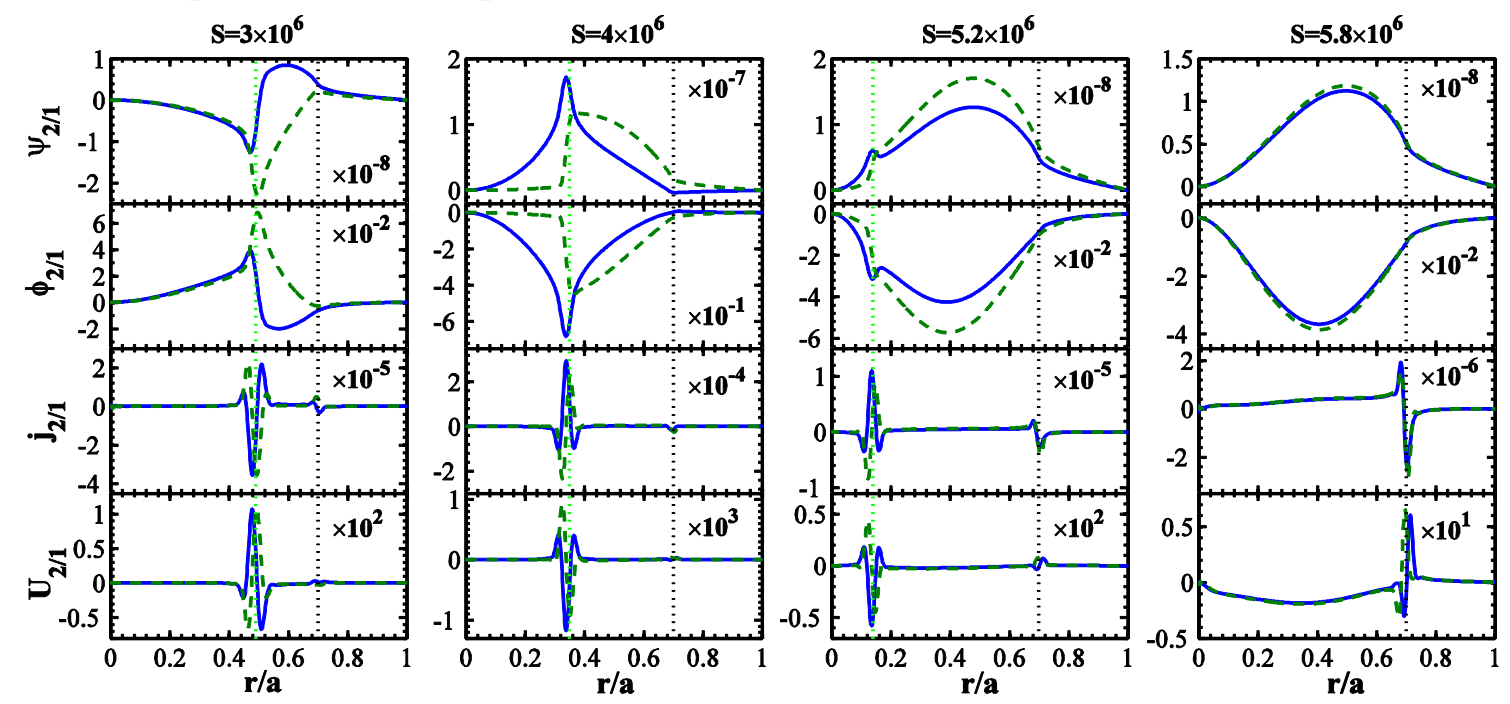

FIG. 5 Corresponding to Fig. $4(\mathrm{c}), \psi_{2 / 1}, \phi_{2 / 1}, j_{2 / 1}$, and $U_{2 / 1}$ for (a) $\mathrm{S}=3 \times 10^{6}$, (b) $\mathrm{S}=4 \times 10^{6}$, (c) $\mathrm{S}=5.2 \times 10^{6}$ and (d) $\mathrm{S}=$ $5.8 \times 10^{6}$. Here, $\omega_{0}=6 \times 10^{4}$, the solid (dash) curve represents the real (imaginary) part. 
Fig. 5 indicates that the radial location of the additional perturbation moves towards $r=0$ with increasing the value of $\mathrm{S}$. It is likely to be caused by Alfvén resonance (AR). ${ }^{13-15} \mathrm{AR}$ is known to occur at a location where $\Delta \omega_{f}=\omega_{\mathrm{A} 1}=\omega_{\mathrm{A}}|m / q-n|$, where $\Delta \omega_{f}=m\left|\omega_{\theta}(r)-\omega_{0}\right|$ is the frequency difference between that at the $q=2$ surface and at the minor radius $r$, and $\omega_{\mathrm{A}}=(\mathrm{m} / \mathrm{r}) V_{\mathrm{A}}$ is the local shear Alfvén frequency ${ }^{13,15}$. Previous studies reported that such ARs can be induced by RMPs ${ }^{13,14}$ and by the perturbations of tearing instabilities. ${ }^{15,26}$

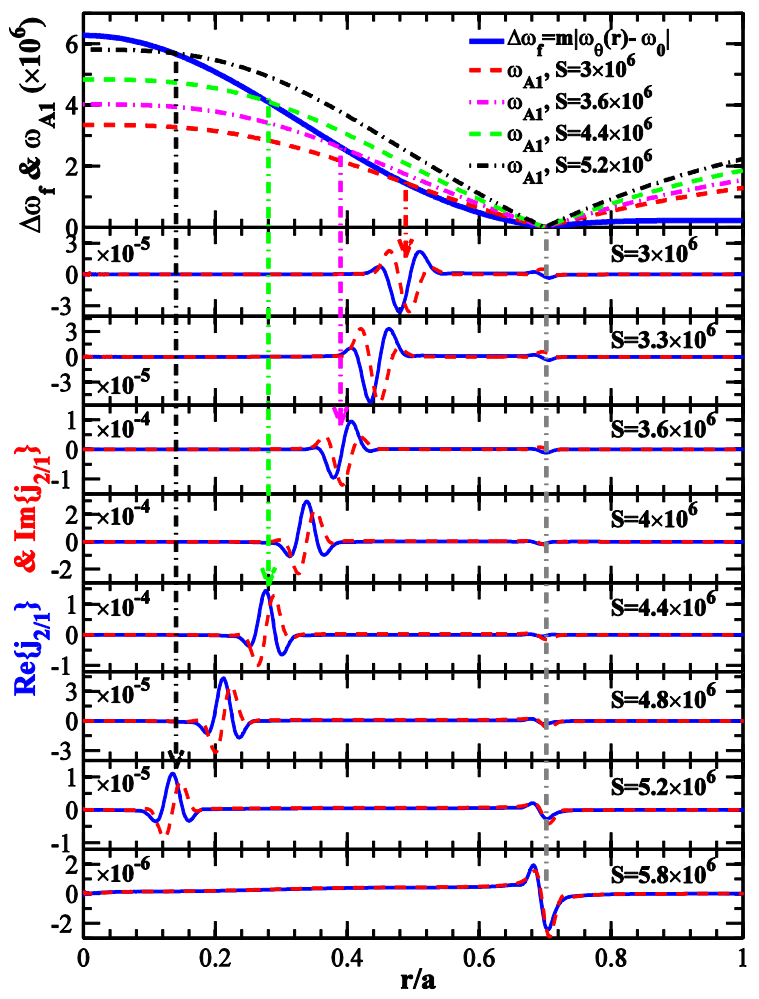

FIG. 6 Corresponding to Fig. 4(c), radial profiles of frequency difference $\Delta \omega_{f}=m\left|\omega_{\theta}(r)-\omega_{0}\right|$, shear Alfvén frequency $\omega_{\mathrm{A} 1}=\omega_{\mathrm{A}}|m / q-n|$, and radial profiles of $j_{2 / 1}$ for different $\mathrm{S}$ are shown. The dot-dashed arrows indicate the locations satisfying the Alfvén resonance condition $\Delta \omega_{f}=\omega_{\mathrm{A} 1}$, and the dot-dashed line indicates the $2 / 1$ rational surface at $r=0.7 \mathrm{a}$.

Corresponding to Figs. 4(c) and 5, radial profiles of $\Delta \omega_{f}$, shear Alfvén wave frequency $\omega_{\mathrm{A} 1}$ and $j_{2 / 1}$ are shown in Fig. 6 for different $S$ values. It is found that for $S<5.8 \times 10^{6}$, the AR condition can be satisfied in the region $0<r<0.7 a$, and the resonance location changes from $r=0.5 a$ to $r=0.14 a$ with the change of $\mathrm{S}$ values. The radial profiles of $j_{2 / 1}$ show that the plasma current density perturbation forms at the location where the AR conditions are satisfied. As the value of $S$ increases, the location of the current density perturbation moves towards $r=0$ until the AR conditions can't be satisfied. In addition, it is found that the amplitude of current density perturbation increases with increasing $\mathrm{S}$ for $\mathrm{S}<4 \times 10^{6}$ but decreases with increasing $\mathrm{S}$ for $4 \times 10^{6}<\mathrm{S}<5.8 \times 10^{6}$. Such a change is the same as that of $\gamma \tau_{\mathrm{A}}$ for the case with $\omega_{0}=6 \times 10^{4}$ as shown in Fig. 4(c). Hence, Figs. 5 and 6 indicate that, the complicated change in $\gamma \tau_{\mathrm{A}}$ for a strong flow shear rate in the low $\mathrm{S}$ regime shown in Fig. 4(c) is caused by AR. A larger current density perturbation results in a larger growth rate.

\section{B. Nonlinear results}

In this section, the effects of sheared plasma flow on nonlinear saturation of the $2 / 1 \mathrm{TM}$ are given. The saturated island width $w / a$ versus $\omega_{0}$ for different flow shear length $L_{\mathrm{v}}$ are shown in Fig. 7 , where $S=1 \times 10^{7}$ and $\mu_{N}=2.8$ are used. For $L_{\mathrm{v}}=2.87$, w/ $a$ decreases with increasing $\omega_{0}$, and the $2 / 1 \mathrm{TM}$ becomes stable if $\omega_{0}>8 \times 10^{4}$. However, when the sheared plasma flow is strong enough, $L_{\mathrm{v}}$ $\leq 0.55$, the mode becomes more unstable and saturates at higher level for a fast plasma flow. Taking the case with $L_{\mathrm{v}}=0.3$ for example, firstly $w / a$ decreases with increasing $\omega_{0}$ for $\omega_{0}<2 \times 10^{4}$ but increases with increasing $\omega_{0}$ for a larger $\omega_{0}$, and the saturated island width increases to 0.16 for $\omega_{0}=$ $8 \times 10^{4}$. When looking back to the linear results as shown in Fig. 4 , it is seen that for $\mathrm{S}=1 \times 10^{7}, \gamma \tau_{\mathrm{A}}$ 
decreases with increasing $\omega_{0}$ for weak shear rate (larger $L_{\mathrm{v}}$ ) but increases with increasing $\omega_{0}$ for strong shear rate $\left(L_{\mathrm{v}} \leq 0.55\right)$ due to the AR. Fig. 7 indicates that when the plasma flow isn't strong enough to be close to AR, a larger plasma flow contributes a stronger stabilizing effect to $2 / 1$ TM, resulting in a lower saturated island width. However, when the plasma flow is strong enough to approach AR, the 2/1 TM saturates at a larger island width. Results shown in Fig. 7 are consistent with the linear result as shown in Fig. 4.

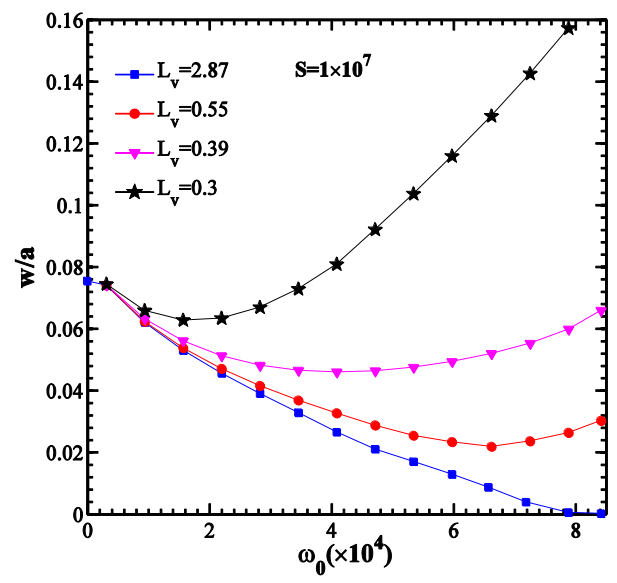

FIG. 7. Normalized saturated island width $w /$ a versus $\omega_{0}$ for different flow shear length with $\mathrm{S}=1 \times 10^{7}$ and $\mu_{N}=2.8$.

Fig. 8(a) shows the normalized saturated island width $w / a$ as a function of $\omega_{0}$ for different $\mathrm{S}$ value and flow shear length $L_{\mathrm{v}}$ with $\mu_{N}=2.8$. As the resistive time is fixed in our calculations, the different $\mathrm{S}$ values correspond to different Alfvén velocity since $V_{\mathrm{A}} \propto \mathrm{S}$. For $\mathrm{S}=2 \times 10^{6}$ (a smaller Alfvén velocity), when the shear rate is weak with $L_{\mathrm{v}}=2.87$, the sheared plasma flow contributes a stabilizing effect to $2 / 1 \mathrm{TM}$, which becomes stable for $\omega_{0}>1.8 \times 10^{4}$. However, when the shear rate is strong enough, $L_{\mathrm{v}}=0.3$, the AR conditions are satisfied for $\omega_{0}>1 \times 10^{4}$, and the $2 / 1 \mathrm{TM}$ saturates at a wider island width. For $\mathrm{S}=5 \times 10^{7}$ (a larger Alfvén velocity), w/a decreases with increasing $\omega_{0}$, and there is no obvious difference in the saturated island width for different $L_{\mathrm{v}}$, since in this case the sheared plasma flow is not strong enough to be close to AR. Further calculations have also been carried out for a larger value of $S, S=1 \times 10^{8}$, and similar results are found. For the plasmas with high value of $S$, the AR condition is usually not satisfied unless the flow is extremely fast, and the sheared plasma flow contributes a stabilizing effect to the TM in this case, which agrees with the observation in DIII-D ${ }^{7}$. In Fig. 8(b), w/a is shown as a function of $\omega_{0}$ for different plasma viscosity and flow shear length $L_{\mathrm{v}}$ with $\mathrm{S}=1 \times 10^{7}$. It reveals that a stronger plasma viscosity makes the $2 / 1$ TM to be more stable. The larger saturated island width for large $\omega_{0}$ with $L_{\mathrm{v}}=0.3$ is also caused by AR.
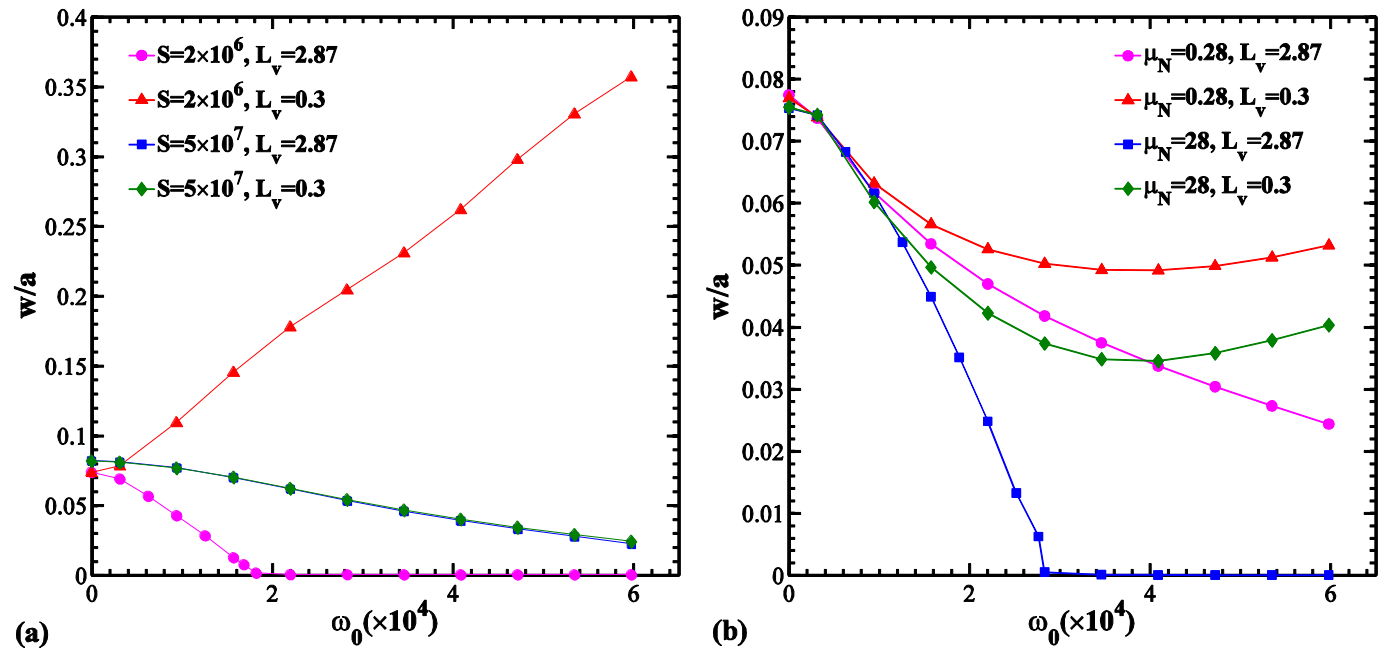

FIG. 8. Saturated island width $w / a$ versus $\omega_{0}$. (a) $\mathrm{S}=2 \times 10^{6}$ and $5 \times 10^{7}, L_{\mathrm{v}}=2.87$ and 0.3 , and $\mu_{N}=2.8$. (b) $\mu_{N}=0.28$ and 
$28, L_{\mathrm{v}}=2.87$ and 0.3 , and $\mathrm{S}=1 \times 10^{7}$.

In Refs. 16 and 17, it has been reported that the flow shear deformed the magnetic island structure and hence stabilized the TM though a more negative classical TM index. For $\mathrm{S}=1 \times 10^{8}$ and $L_{\mathrm{v}}=0.3$, the contour plots of magnetic flux for $\omega_{0}=3.2 \times 10^{3}$ and $\omega_{0}=6 \times 10^{4}$ are shown in Fig. 9, respectively. For a weak plasma flow as shown in Fig. 9(a), the island saturates with $w / a=0.081$. For a strong plasma flow as shown in Fig. 9(b), the island saturates with a much smaller width (w/a $=0.011)$. However, no significant distortion in the island structure is observed.

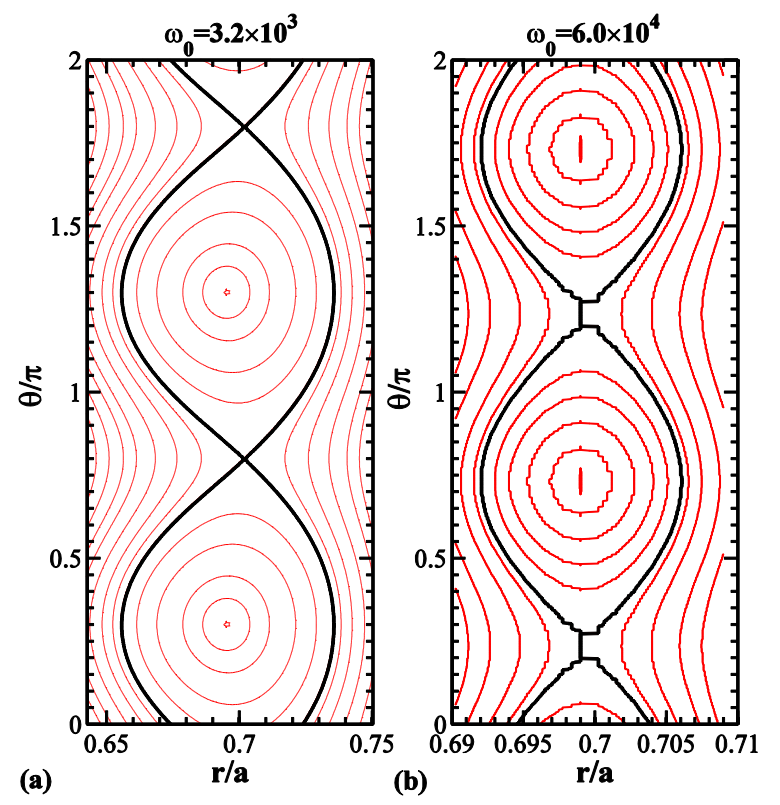

FIG. 9. Corresponding to Fig.7(b), the contour plot of magnetic flux for (a) $\omega_{0}=3.2 \times 10^{3}$ and (b) $\omega_{0}=6 \times 10^{4}$. Here the flow shear length $L_{\mathrm{v}}=0.3$.
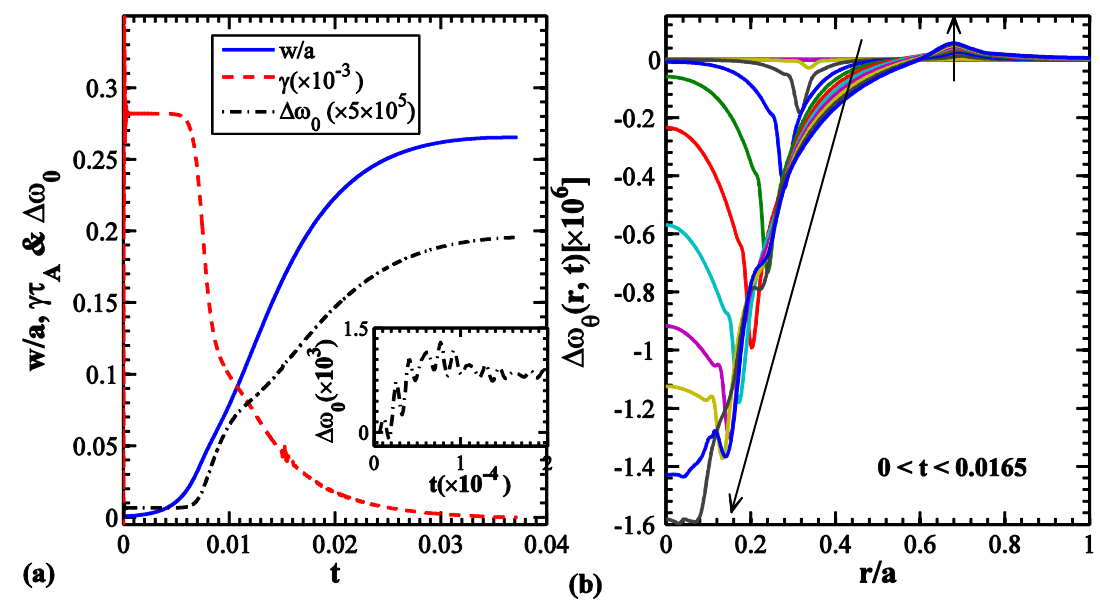

FIG. 10. Corresponding to the case with $\omega_{0}=6 \times 10^{4}$ and $S=4 \times 10^{6}$ (the peak point) in Fig.4(c), (a) the nonlinear evolution of island width $w / a$, growth rate $\gamma \tau_{\mathrm{A}}$ and $\Delta \omega_{0}$, here $\Delta \omega_{0}(t)=\omega_{0}(t)-\omega_{0}(t=0)$. (b) Evolution of radial profiles of $\Delta \omega_{\theta}$ for $0<t / \tau_{\mathrm{R}}<0.0165$, here $\Delta \omega_{\theta}(r, t)=\omega_{\theta}(r, t)-\omega_{\theta}(r, t=0)$, the arrows indicates the trend of changes. The detail evolution of $\Delta \omega_{0}$ at the beginning is shown at the lower right corner of Fig. 10(a).

Corresponding to the case with $\omega_{0}=6 \times 10^{4}$ and $S=4 \times 10^{6}$ (the peak point) in Fig.4(c), the nonlinear evolution of $2 / 1$ island width $w / a$, growth rate $\gamma \tau_{\mathrm{A}}$ and $\Delta \omega_{0}$ are shown in Fig. 10(a), where the relative change of the mode angular frequency $\Delta \omega_{0}(t)=\omega_{0}(t)-\omega_{0}(t=0)$. In the linear phase, both $\gamma \tau_{\mathrm{A}}$ and $\Delta \omega_{0}$ are constant, and it need to note that $\Delta \omega_{0}$ doesn't equal 0 in the linear phase because the adjustment of equilibrium, as indicated by detail evolution of $\Delta \omega_{0}$ at the lower right corner of Fig. 10(a). In the nonlinear stage, $\gamma \tau_{\mathrm{A}}$ decreases, and the island width saturates at $w / a=0.27$. In this phase $\Delta \omega_{0}$ increases substantially, indicating considerable increase in the plasma rotation at the $2 / 1$ 
rational surface In Fig. 10(b), the time evolution of radial profiles of $\Delta \omega_{\theta}$ are shown for $0<t / \tau_{\mathrm{R}}<$ 0.0165 , where $\Delta \omega_{\theta}(r, t)=\omega_{\theta}(r, t)-\omega_{\theta}(r, t=0)$. It is found that during the nonlinear stage, the amplitude of $\Delta \omega_{\theta}$ increases around the location of AR, and the change of $\Delta \omega_{\theta}$ spreads towards the plasma core $(r=0)$. The location of peak point of $\Delta \omega_{\theta}$ changes from $r=0.34 a$ (the original AR position) to $r=0.1 a$. Meanwhile, $\Delta \omega_{\theta}$ increases around the $2 / 1$ rational surface.

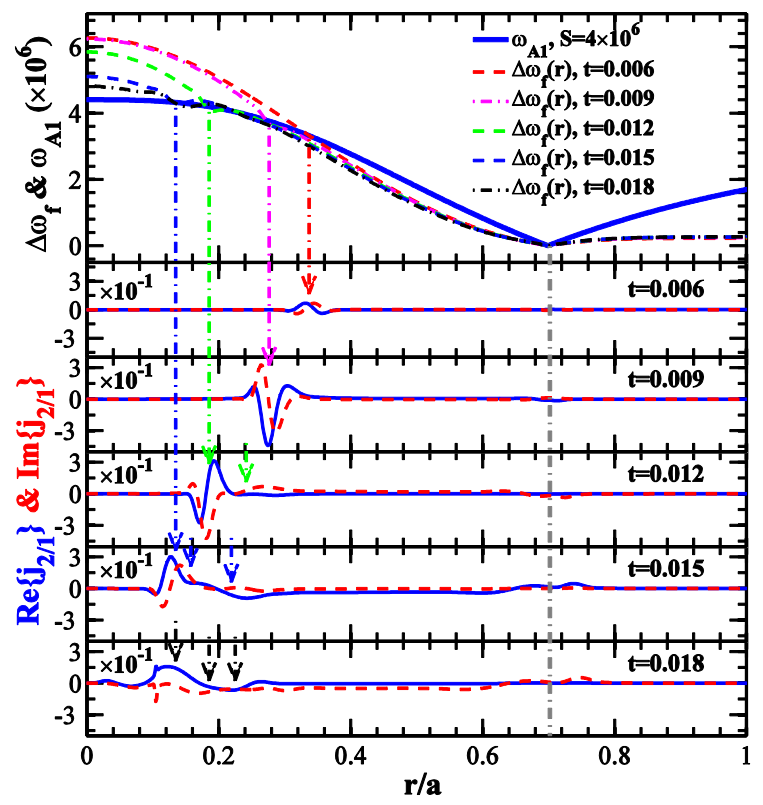

FIG. 11 Corresponding to Fig. 10, radial profiles shear Alfvén wave frequency $\omega_{\mathrm{A} 1}=\omega_{\mathrm{A}}|m / q-n|$ for $\mathrm{S}=4 \times 10^{6}$ and $\Delta \omega_{f}(r)$ at different times. The dot-dashed arrows indicate the locations satisfying the Alfvén resonance condition $\Delta \omega_{f}=\omega_{\mathrm{A} 1}$, and the dot-dashed line indicates the 2/1 rational surface.
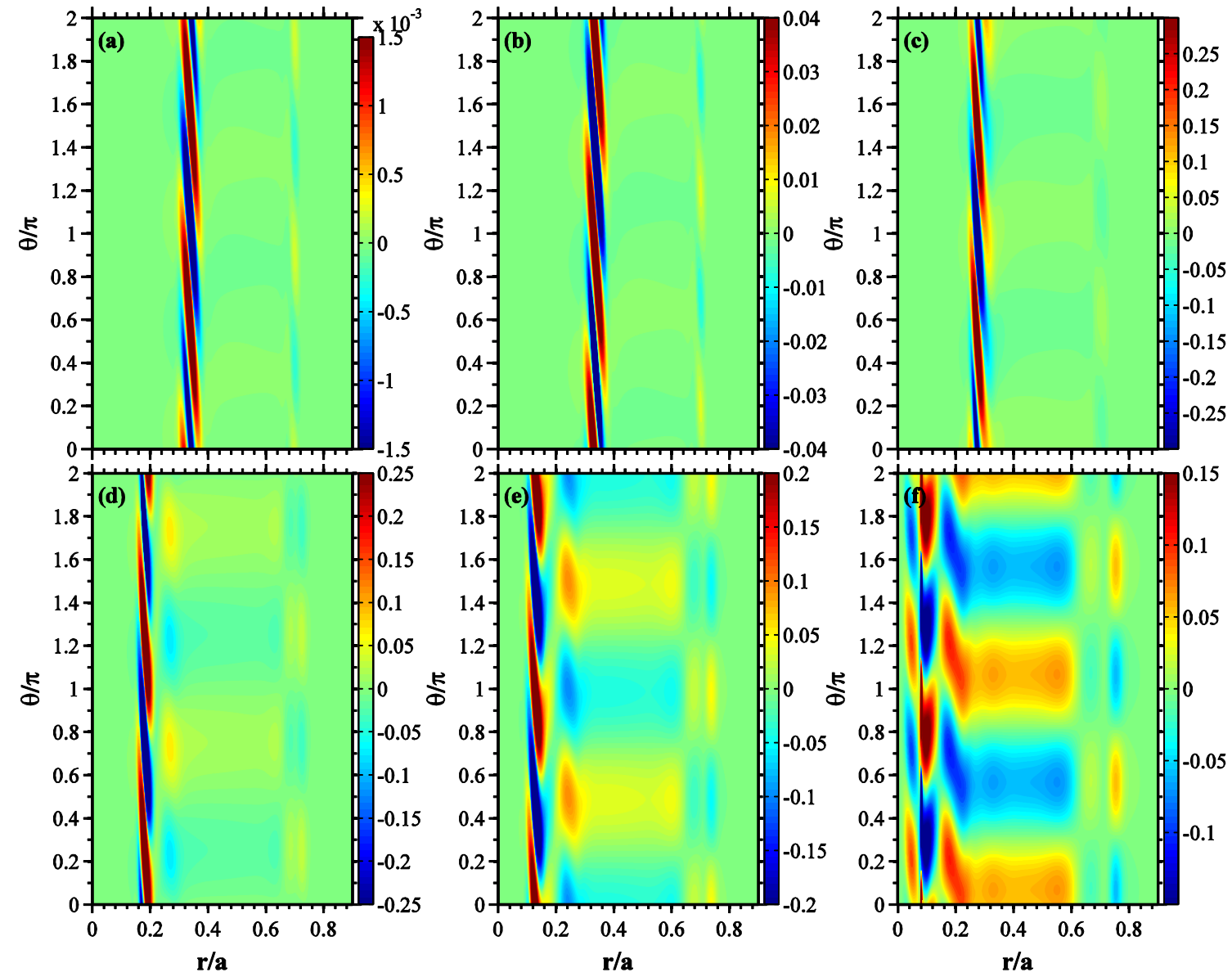

FIG. 12 Corresponding to Fig. 10, contour plots of the $2 / 1$ component of perturbed current density $j_{2 / 1}$ for (a) $t=0.003$, (b) $t=0.006$, (c) $t=0.009$, (d) $t=0.012$, (e) $t=0.015$ and (f) $t=0.018$. 
For a constant shear Alfvén frequency $\omega_{\mathrm{A}}$, the radial location of AR will change when the plasma rotation frequency changes as shown in Fig. 10(b). In Fig. 11 the radial profiles of $\omega_{\mathrm{A}}, \Delta \omega_{f}(r)$ and the corresponding $j_{2 / 1}$ at different times are shown. In the linear stage, for $t=0.006, \omega_{\theta}(r)$ changes little and the AR position is at $r=0.34 a$. In the nonlinear stage, accompanying with the decrease of $\omega_{\theta}(r)$ in the central region, the AR location moves inwards, being at $r=0.28 a$ for $t=$ $0.009, r=0.19 a$ for $t=0.012, r=0.13 a$ for $t=0.015$, and $r=0.12 a$ for $t=0.018$. Besides, the amplitude of $j_{2 / 1}$ at AR increases during the time period of $0.006<t<0.012$. However, for $t \geq 0.012$, because of the substantial decrease of $\omega_{\theta}(r)$ in the core region, there are three nearby ARs as marked by minor arrows at $t=0.012,0.015$ and 0.018 . As a result, the amplitude of $j_{2 / 1}$ at AR is smaller. Corresponding to Fig. 11, in Fig. 12(a)-(f) the contour plots of $j_{2 / 1}$ at $t=0.003,0.006,0.009,0.012$, 0.015 and 0.018 are shown. Fig. 12(a)-(d) clearly show the inward spread of current density associated with the AR, while Fig. 12(d)-(f) shows the diffusion of AR current to global current density perturbations.

\section{Summary}

Using the reduced MHD equations, the effect of sheared plasma flow on tearing stability is studied under the large aspect-ratio and the cold ion approximations. Comparing with previous theoretical works on shear flow, ${ }^{8,9,16}$ the new features of our model are the using of more realistic radial profiles of plasma rotation frequency and higher $S$ values $\left(S \sim 10^{6 \sim 9}\right)$. Only in Refs. 12 and 18 a sheared velocity profile is included in the numerical results. In Ref. 26, numerical results are presented on the effect of shear plasma rotation on the linear mode growth, but the nonlinear effect is neglected.

From the numerical calculations we find that, weak or moderate sheared plasma flow plays a stabilizing effect on 2/1 TM. However, the mode becomes more unstable for a sufficiently strong shear flow in low $S$ regime because of approaching AR (i.e. $S \sim 1 \times 10^{7}$ for J-TEXT ohmic plasma ${ }^{21-22}$ and $S \sim 1 \times 10^{8}$ for DIII-D H-mode plasma ${ }^{5-7}$ ). Similarly, in the nonlinear phase sheared plasma flow leads to a smaller (larger) saturated island width for a moderate (strong) shear plasma flow. For weak or moderate sheared plasma flows, the stabilization of TM by plasma flow is enhanced by a stronger viscosity. In addition, the nonlinear evolution of AR is studied. It is found that AR accelerates the plasma rotation around the 2/1 rational surface while decelerates the plasma rotation at the AR location. As a result, the radial location satisfying AR spreads inwards towards the magnetic axis, and ARs can occurs simultaneously at more than one radial locations, which in turn causes the radial diffusion of the AR current density.

For the plasma rotation frequency discussed in this paper, the angular frequency at the $2 / 1 \mathrm{RS}$ satisfies $\omega_{0} / \omega_{\mathrm{A}}<10^{-2}$. However, depending on the shear rate, the angular frequency at plasma core can be much higher with $\omega_{\theta}(r=0) \sim 10^{6}$, resulting in the AR conditions being satisfied easily for plasma with lower $S$ value $\left(S \sim 10^{6}\right)$. It should be noted that for actual tokamak plasma $\left(S \sim 10^{7-8}\right)$, the actual angular rotation frequency at plasma core ranges around $\sim 10^{5} \mathrm{rad} / \mathrm{s}$ and $\omega_{0}$ ranges from $10^{4} \sim 10^{5} \mathrm{rad} / \mathrm{s}$, being much lower than the shear Alfven frequency. Hence, the flow shear strong enough to satisfy AR conditions is not realistic, and the AR conditions are hard to be satisfied in actual tokamak plasma.

In conclusion, our results indicate that, for plasmas with high $S$ values, the sheared plasma flow usually contributes a stabilizing effect on tearing stability, since the AR condition is not satisfied.

\section{Acknowledgments}

One of the authors (Q. Hu) is grateful to Dr. Lai Wei for fruitful discussion. This work is 
supported by the Ministry of Science and Technology (Contract NO. 2011GB109001 and 2014GB118000) and National Natural Science Foundation (Contract No. 10990214).

\section{References}

M. S. Chu, L. Chen, L. J. Zheng, C. Ren, A. Bondeson, Nuclear Fusion 39, 2107 (1999).

2 M. Keilhacker, J. E. T. T. the, Plasma Physics and Controlled Fusion 41, B1 (1999).

3 M. Ono, S. M. Kaye, Y. K. M. Peng, G. Barnes, W. Blanchard, M. D. Carter, J. Chrzanowski, L. Dudek, R. Ewig, D. Gates, R. E. Hatcher, T. Jarboe, S. C. Jardin, D. Johnson, R. Kaita, M. Kalish, C. E. Kessel, H. W. Kugel, R. Maingi, R. Majeski, J. Manickam, B. McCormack, J. Menard, D. Mueller, B. A. Nelson, B. E. Nelson, C. Neumeyer, G. Oliaro, F. Paoletti, R. Parsells, E. Perry, N. Pomphrey, S. Ramakrishnan, R. Raman, G. Rewoldt, J. Robinson, A. L. Roquemore, P. Ryan, S. Sabbagh, D. Swain, E. J. Synakowski, M. Viola, M. Williams, J. R. Wilson, Nuclear Fusion 40, 557 (2000).

4 J. L. Luxon, Nuclear Fusion 42, 614 (2002).

$5 \quad$ R. J. La Haye, R. J. Buttery, Physics of Plasmas 16, 022107 (2009).

6 R. J. La Haye, D. P. Brennan, R. J. Buttery, S. P. Gerhardt, Physics of Plasmas 17, 056110 (2010).

$7 \quad$ R. J. L. Haye, C. C. Petty, P. A. Politzer, Nuclear Fusion 51, 053013 (2011).

8 X. L. Chen, P. J. Morrison, Physics of Fluids B 2, 495 (1990).

$9 \quad$ L. Ofman, X. L. Chen, P. J. Morrison, R. S. Steinolfson, Physics of Fluids B 3, 1364 (1991).

10 A. Bierwage, Q. Yu, S. Gunter, Physics of Plasmas 14, 010704 (2007).

11 I. T. Chapman, S. Brown, R. Kemp, N. R. Walkden, Nuclear Fusion 52, 042005 (2012).

12 L. Wei , Z. Wang, Physics of Plasmas 20, 012512 (2013).

13 X. Wang, A. Bhattacharjee, Z. W. Ma, C. Ren, C. C. Hegna, J. D. Callen, Physics of Plasmas 5, 2291 (1998).

14 Y. Liu, J. W. Connor, S. C. Cowley, C. J. Ham, R. J. Hastie, T. C. Hender, Physics of Plasmas 19, 102507 (2012).

15 L. Wei, Z. Wang, Plasma Physics and Controlled Fusion 55, 085004 (2013).

16 L. Ofman, P. J. Morrison, R. S. Steinolfson, Physics of Fluids B 5, 376 (1993).

17 C. Ren, M. S. Chu, J. D. Callen, Physics of Plasmas 6, 1203 (1999).

18 D. Chandra, A. Sen, P. Kaw, M. P. Bora, S. Kruger, Nuclear Fusion 45, 524 (2005).

19 R. Coelho, E. Lazzaro, Physics of Plasmas 14, 012101 (2007).

20 T. C. Hender, J. C. Wesley, J. Bialek, A. Bondeson, A. H. Boozer, R. J. Buttery, A. Garofalo, T. P. Goodman, R. S. Granetz, Y. Gribov, O. Gruber, M. Gryaznevich, G. Giruzzi, S. Günter, N. Hayashi, P. Helander, C. C. Hegna, D. F. Howell, D. A. Humphreys, G. T. A. Huysmans, A. W. Hyatt, A. Isayama, S. C. Jardin, Y. Kawano, A. Kellman, C. Kessel, H. R. Koslowski, R. J. L. Haye, E. Lazzaro, Y. Q. Liu, V. Lukash, J. Manickam, S. Medvedev, V. Mertens, S. V. Mirnov, Y. Nakamura, G. Navratil, M. Okabayashi, T. Ozeki, R. Paccagnella, G. Pautasso, F. Porcelli, V. D. Pustovitov, V. Riccardo, M. Sato, O. Sauter, M. J. Schaffer, M. Shimada, P. Sonato, E. J. Strait, M. Sugihara, M. Takechi, A. D. Turnbull, E. Westerhof, D. G. Whyte, R. Yoshino, H. Zohm, Nuclear Fusion 47, S128 (2007).

21 Q. Hu, Q. Yu, B. Rao, Y. Ding, X. Hu, G. Zhuang, Nuclear Fusion 52, 083011 (2012).

22 Q. Hu, B. Rao, Q. Yu, Y. Ding, G. Zhuang, W. Jin, X. Hu, Physics of Plasmas 20, 092502 (2013).

23 Q. Yu, S. Gunter, K. Lackner, Physics of Plasmas 11, 140 (2004).

24 Q. Yu, S. Gunter, Nuclear Fusion 48, 065004 (2008).

25 H. P. Furth, J. Killeen, M. N. Rosenbluth, Physics of Fluids 6, 459 (1963).

26 L. Wei, Z. Wang, Nuclear Fusion 51, 123005 (2011). 